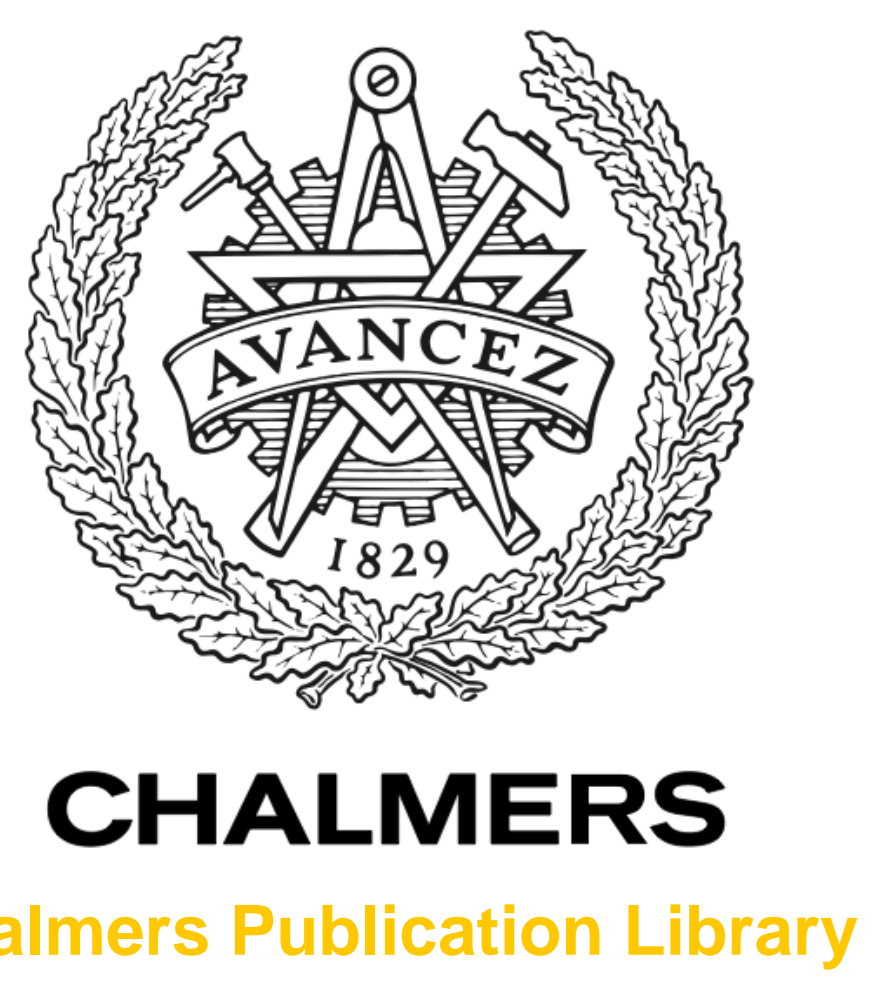

Chalmers Publication Library

\title{
Channel Tracking for AF MIMO Relaying Systems
}

This document has been downloaded from Chalmers Publication Library (CPL). It is the author's version of a work that was accepted for publication in:

Proceedings of the 76th Vehicular Technology Conference, VTC Fall 2012, SEP 03-06

Citation for the published paper:

Lioliou, P. ; Svensson, D. ; Viberg, M. (2012) "Channel Tracking for AF MIMO Relaying Systems". Proceedings of the 76th Vehicular Technology Conference, VTC Fall 2012, SEP 03-06

Downloaded from: http://publications.lib.chalmers.se/publication/166388

Notice: Changes introduced as a result of publishing processes such as copy-editing and formatting may not be reflected in this document. For a definitive version of this work, please refer to the published source. Please note that access to the published version might require a subscription. 


\title{
Channel Tracking for AF MIMO Relaying Systems
}

\author{
Panagiota Lioliou, Daniel Svensson, and Mats Viberg \\ Department of Signals and Systems, Chalmers University of Technology, Gothenburg, Sweden \\ E-mail:\{panagiota.lioliou, daniel.svensson, viberg\}@ chalmers.se
}

\begin{abstract}
In this paper, we consider the problem of channel estimation in multiple-input multiple-output (MIMO) amplifyand-forward (AF) relaying systems operating over time varying channels. Only data at the receiving end are assumed available for the estimation. By employing a first-order autoregressive (AR) model for characterizing the time-varying nature of the channels to be estimated, we derive an expectation-maximization (EM) Kalman filter (KF) that utilizes the received signal at the destination to track the individual channel links. The extended $\mathrm{KF}$ algorithm is also derived and compared to the proposed EM-based KF. Our simulation results show that the proposed EM-based KF offers better estimation performance with less complexity when compared to the EKF algorithm.
\end{abstract}

\section{INTRODUCTION}

Multiple-input multiple-output (MIMO) wireless communications and relaying technology have recently become the focus of extensive research studies. The deployment of relaying nodes in wireless networks has been identified as a promising technique that can offer a number of significant performance benefits, including broader coverage, higher transmission rates, and increased reliability [1]. Moreover, when the source and destination are equipped with multiple antennas, the system's performance can be further enhanced by exploiting the spatial dimension [2].

Several authors have studied and analyzed the performance of relaying systems in terms of their outage probability and end-to-end signal-to-noise ratio (SNR) [3]. Moreover, several designs for the optimal relay amplifying factor have been proposed for maximizing the received SNR or some other performance measure, such as the channel capacity [4]. The aforementioned techniques, as well as most existing relaying schemes, require accurate channel state information (CSI) at the receiver, and sometimes, at the relays as well.

Despite the importance of this prerequisite, channel estimation is often ignored by either assuming perfect CSI or by considering only the estimation of the compound (from source to destination) channel [5]. While CSI of the compound channel guarantees feasible data detection at the destination, the knowledge of the individual channel responses can be utilized to further improve the overall system performance. Under the assumption that the channels are fixed during a long enough time interval, the individual channels can be estimated by using the algorithms proposed in [6], [7]. However, in practice, the channels may vary with time due to the mobility of the receiver and/or the relaying nodes. In these cases, the implementation of estimation algorithms that can track the channel variations becomes essential.

Motivated by this observation, we hereafter consider the problem of channel estimation in MIMO AF relaying sys- tems with time varying channels. While the authors in [8] propose a Kalman filter (KF) based algorithm for estimating only the compound channel at the destination, we take one step further and construct the problem of channel estimation such that all channel parameters involved in the transmission are estimated by using measurements only at the destination. In particular, we derive an expectation-maximization (EM) method for tracking the individual channel responses. By employing a first order-autoregressive (AR) model for characterizing the time-varying nature of the channels to be estimated, the proposed algorithm boils down to an EM-based $\mathrm{KF}$ that utilizes the received signal at the destination to track the individual channel links. Moreover, an alternative channel tracking technique based on the extended KF algorithm [9] is derived and compared to the proposed EM-based KF.

Notation. We use bold upper-case letters to denote matrices and bold lower-case letters to denote vectors. The transpose, Hermitian, and inverse of a matrix $\mathbf{A}$ are denoted by $\mathbf{A}^{T}$, $\mathbf{A}^{*}$, and $\mathbf{A}^{-1}$, respectively; $\mathbf{I}_{N}$ is the identity matrix of size $N$. With $\operatorname{vec}(\mathbf{A})$, we denote the vectorization of a matrix $\mathbf{A}$; $\operatorname{Tr}(\mathbf{A})$ is the trace of the matrix $\mathbf{A}, \operatorname{blkdiag}\{\cdot\}$ stands for a block-diagonal matrix, while $E\{\cdot\}$ denotes expectation.

\section{System And Signal Model}

We consider the downlink relay channel from a fixed source (S) to a mobile destination node (D) via $R$ intermediate relaying nodes. The source and the destination are equipped with $N$ and $M$ antennas, respectively, whereas the relaying nodes are equipped with one antenna each. Let us denote the complex channel matrix from source to relays during the $k$ th transmission block as $\mathbf{H}_{1, k} \in \mathcal{C}^{R \times N}$, and from relays to destination as $\mathbf{H}_{2, k} \in \mathcal{C}^{M \times R}$. We assume that the channels remain fixed within one block of length $L$ but can change between different blocks. Moreover, it is assumed that there is no direct path between $\mathrm{S}$ and $\mathrm{D}$ due to the surrounding environment (e.g in an urban environment).

Based on the above assumptions, the $k$ th received block at the destination can be written as

$$
\mathbf{Y}_{k}=\mathbf{H}_{2, k} \mathbf{G}_{k} \mathbf{H}_{1, k} \mathbf{X}_{k}+\mathbf{H}_{2, k} \mathbf{G}_{k} \mathbf{W}_{R, k}+\mathbf{W}_{D, k}
$$

where $\mathbf{X}_{k} \in \mathcal{C}^{N \times L}$ is the matrix of transmitted signals, $\mathbf{G}_{k} \in \mathcal{C}^{R \times R}$ is the relay amplification matrix, while $\mathbf{w}_{R, k} \sim$ $\mathcal{C N}\left(0, \sigma_{r}^{2} \mathbf{I}_{R}\right)$ and $\mathbf{w}_{D, k} \sim \mathcal{C N}\left(0, \sigma_{d}^{2} \mathbf{I}_{M}\right)$ are the independent white complex Gaussian noise contributions at the relays and the destination, respectively. If we assume that the relays operate in a distributed manner, then $\mathbf{G}_{k} \in \mathcal{C}^{R \times R}$ is diagonal, and its diagonal elements contain the complex amplifying 
factors, $\left\{g_{i, k}\right\}_{i=1}^{R}$, for each relaying node. We can now rewrite (1) as

$$
\mathbf{Y}_{k}=\mathbf{H}_{k} \mathbf{X}_{k}+\mathbf{W}_{k},
$$

where $\mathbf{H}_{k}=\mathbf{H}_{2, k} \mathbf{G}_{k} \mathbf{H}_{1, k}$ and $\mathbf{W}_{k}=\mathbf{H}_{2, k} \mathbf{G}_{k} \mathbf{W}_{R, k}+\mathbf{W}_{D, k}$ correspond to the equivalent (compound) channel matrix and noise, respectively.

In this work, we adopt a first-order autoregressive (AR) model to characterize the time-varying behavior of the individual channel responses, since it accurately captures the dynamics of the wireless channel, while remaining mathematically tractable [10]. Therefore, the state evolution of the channel vectors $\mathbf{h}_{1, k}=\operatorname{vec}\left(\mathbf{H}_{1, \mathrm{k}}\right)$ and $\mathbf{h}_{2, k}=\operatorname{vec}\left(\mathbf{H}_{2, \mathrm{k}}\right)$ can be described by the following first-order models

$$
\mathbf{h}_{1, k}=\mathbf{A} \mathbf{h}_{1, k-1}+\mathbf{v}_{k}, \mathbf{h}_{2, k}=\mathbf{B h}_{2, k-1}+\mathbf{u}_{k},
$$

where $\mathbf{A}=\alpha \mathbf{I}_{\mathbf{R}}, \mathbf{B}=\beta \mathbf{I}_{\mathbf{M}}, \alpha$ and $\beta$ are the static AR coefficients, while $\mathbf{v}_{k} \sim \mathcal{C N}\left(0, \sigma_{v}^{2} \mathbf{I}_{N R}\right)$ and $\mathbf{u}_{k} \sim \mathcal{C N}\left(0, \sigma_{u}^{2} \mathbf{I}_{M R}\right)$ are the complex driving noises of the models. We further assume that $\mathbf{H}_{1, k}$ is a fixed-to-mobile channel while $\mathbf{H}_{2, k}$ is a mobile-to-mobile channel. The discrete autocorrelation functions of $\mathbf{H}_{1, k}$ and $\mathbf{H}_{2, k}$ can be expressed as [10]

$$
\begin{gathered}
E\left\{\mathbf{h}_{1,\left(k+k^{\prime}\right)} \mathbf{h}_{1, k}^{*}\right\}=J_{0}\left(2 \pi f_{D 1} k^{\prime}\right) \mathbf{I}_{N R}, \\
E\left\{\mathbf{h}_{2,\left(k+k^{\prime}\right)} \mathbf{h}_{2, k}^{*}\right\}=J_{0}\left(2 \pi f_{D 1} k^{\prime}\right)\left(2 \pi f_{D 2} k^{\prime}\right) \mathbf{I}_{M R},
\end{gathered}
$$

where $f_{D 1}$ and $f_{D 2}$ are the maximum Doppler frequencies due to the motion of the relays and the destination, respectively. The AR coefficients in (3) can then be calculated as

$$
\alpha=J_{0}\left(2 \pi f_{D 1} T_{s}\right), \quad \beta=J_{0}\left(2 \pi f_{D 1} T_{s}\right) J_{0}\left(2 \pi f_{D 2} T_{s}\right),
$$

where $1 / T_{s}$ is the sampling rate.

\section{Kalman-Based Channel Tracking}

In this section, we study the problem of channel tracking for AF relaying systems. For the sake of simplicity, we initially study the case of one relay $(R=1)$ and then move onto the more general case of $R>1$.

In order to formulate the problem of jointly tracking the individual channel responses $\mathbf{h}_{1, k} \in \mathcal{C}^{N \times 1}$ and $\mathbf{h}_{2, k} \in \mathcal{C}^{M \times 1}$ at the destination, we need a process and a measurement model. Since the process model describes the dynamic behavior of the state variables, we can regard (3) as the process (state-space) equations, while the measurement (observation) equation is described in (1). After vectorizing each term in (1), and using the identity vec $(\mathbf{A B C})=\left(\mathbf{C}^{T} \otimes \mathbf{A}\right) \operatorname{vec}(\mathbf{B})$, we can rewrite (1) as

$$
\mathbf{y}_{k}=\left(\left(g_{k} \mathbf{h}_{1, k}^{T} \mathbf{X}_{k}\right)^{T} \otimes \mathbf{I}_{M}\right) \mathbf{h}_{2, k}+\mathbf{w}_{k},
$$

where $g_{k}$ is the relay amplification factor, and $\mathbf{w}_{k}=\operatorname{vec}\left(\mathbf{W}_{\mathbf{k}}\right)$ is the vectorized form of the compound noise at the destination with covariance matrix $\mathbf{R}_{\mathrm{w}}=\left(\sigma_{r}^{2} g_{k} g_{k}^{*}+\sigma_{d}^{2}\right) \mathbf{I}_{M L}$. Equivalently, (7) can also be expressed as

$$
\mathbf{y}_{k}=\left(\mathbf{X}_{k}^{T} \otimes \mathbf{h}_{2, k} g_{k}\right) \mathbf{h}_{1, k}+\mathbf{w}_{k} .
$$

Note that while the state-space equations in (3) are linear, the observation equations described by (7) and (8), are nonlinear functions of the unknown channel vectors $\mathbf{h}_{1, k}$ and $\mathbf{h}_{2, k}$ thereby precluding the direct application of the Kalman filter (KF). Motivated by this observation, in the following we develop extended KF (EKF) and expectation-maximization (EM) based KF algorithms for tracking the individual channel responses at the destination.

\section{A. EM-based Kalman Filter}

Ideally, we could jointly estimate $\mathbf{h}_{1, k}$ and $\mathbf{h}_{2, k}$ using some pilot-output relationship, e.g. (8), by maximizing the corresponding unnormalized posterior distribution function

$$
\left\{\hat{\mathbf{h}}_{1, k}, \hat{\mathbf{h}}_{2, k}\right\}=\arg \max _{\mathbf{h}_{1, k}, \mathbf{h}_{2, k}} \log p\left(\mathbf{h}_{1, k}, \mathbf{h}_{2, k} \mid \mathbf{y}_{k}\right) .
$$

After straightforward calculations, we can arrive at the following expression for the log-posterior $\mathcal{L}\left(\mathbf{h}_{1, k}, \mathbf{h}_{2, k} \mid \mathbf{y}_{k}\right)=$ $\log p\left(\mathbf{h}_{1, k}, \mathbf{h}_{2, k} \mid \mathbf{y}_{k}\right)$ (up to some additive constant)

$$
\begin{aligned}
\mathcal{L}\left(\mathbf{h}_{1, k}, \mathbf{h}_{2, k}\right)= & -\left\|\mathbf{y}_{k}-\tilde{\mathbf{H}}_{1, k} \mathbf{h}_{2, k}\right\|_{\mathbf{R}_{\mathbf{w}}^{-1}}^{2} \\
& -\left\|\mathbf{h}_{1, k}-\mathbf{A} \mathbf{h}_{1, k-1}\right\|_{1 / \sigma_{v}^{2}}^{2}-\left\|\mathbf{h}_{1,0}\right\|^{2} \\
& -\left\|\mathbf{h}_{2, k}-\mathbf{B} \mathbf{h}_{2, k-1}\right\|_{1 / \sigma_{u}^{2}}^{2}-\left\|\mathbf{h}_{2,0}\right\|^{2},
\end{aligned}
$$

where $\tilde{\mathbf{H}}_{1, k}=\left(g_{k} \mathbf{h}_{1, k}^{T} \mathbf{X}_{k}\right)^{T} \otimes \mathbf{I}_{M}$. By inspecting (10), we note that the optimization problem in (9) is nonlinear and nonconvex. In other words, closed-form solutions for the optimal maximum-a-posteriori (MAP) estimates $\hat{\mathbf{h}}_{1, k}$ and $\hat{\mathbf{h}}_{2, k}$ cannot be derived. To overcome this limitation, we apply the EM algorithm, and maximize the log-posterior function averaged over $\mathbf{h}_{1, k}$ (treated as the missing data) [11]. Specifically, starting from an initial estimate $\hat{\mathbf{h}}_{2, k}^{(0)}$, the estimate $\mathbf{h}_{2, k}$ is calculated iteratively, with the estimate at the $j$ th iteration given by

$$
\hat{\mathbf{h}}_{2, k}^{(j)}=\arg \max _{\mathbf{h}_{2, k}}\left\{E_{\mathbf{h}_{1, k} \mid \mathbf{y}_{k}, \hat{\mathbf{h}}_{2, k}^{(j-1)}} \mathcal{L}\left(\mathbf{h}_{1, k}, \mathbf{h}_{2, k}\right)\right\}
$$

where the expectation is taken w.r.t $\mathbf{h}_{1, k}$, given the most recent estimate $\hat{\mathbf{h}}_{2, k}^{(j-1)}$ and the output sequence $\mathbf{y}_{k}$. From (10), we note that only the first term is modified under expectation and its expectation can be evaluated as

$$
\begin{aligned}
E\left\|\mathbf{y}_{k}-\tilde{\mathbf{H}}_{1, k} \mathbf{h}_{2, k}\right\|_{\mathbf{R}_{\mathbf{w}}^{-1}}^{2}= & \left\|\mathbf{y}_{k}-E\left\{\tilde{\mathbf{H}}_{1, k}\right\} \mathbf{h}_{2, k}\right\|_{\mathbf{R}_{\mathbf{w}}^{-1}}^{2} \\
& +\left\|\mathbf{h}_{2, k}\right\|_{\mathbf{R}_{\mathbf{w}}^{-1} \operatorname{Cov}\left\{\tilde{\mathbf{H}}_{1, k}^{*}\right\}}^{2}
\end{aligned}
$$

Thus, the averaged log-function $\tilde{\mathcal{L}}\left(\mathbf{h}_{1, k}, \mathbf{h}_{2, k}\right)$ is expressed as

$$
\begin{aligned}
\tilde{\mathcal{L}}\left(\mathbf{h}_{1, k}, \mathbf{h}_{2, k}\right)= & \left\|\left[\begin{array}{c}
\mathbf{y}_{k} \\
\mathbf{0}_{R M \times 1}
\end{array}\right]-\left[\begin{array}{c}
E\left\{\tilde{\mathbf{H}}_{1, k}\right\} \\
\operatorname{Cov}\left\{\tilde{\mathbf{H}}_{1, k}^{*}\right\}^{1 / 2}
\end{array}\right]\right\|_{\mathbf{R}_{\mathbf{w}}^{-1}}^{2} \\
& -\left\|\mathbf{h}_{1, k}-\mathbf{A h}_{1, k-1}\right\|_{1 / \sigma_{v}^{2}}^{2}-\left\|\mathbf{h}_{1,0}\right\|^{2} \\
& -\left\|\mathbf{h}_{2, k}-\mathbf{B h}_{2, k-1}\right\|_{1 / \sigma_{u}^{2}}^{2}-\left\|\mathbf{h}_{2,0}\right\|^{2} .
\end{aligned}
$$

Note that one can obtain (13) from the original function (10) by performing the substitution

$$
\tilde{\mathbf{H}}_{1, k} \rightarrow\left[\begin{array}{c}
E\left\{\tilde{\mathbf{H}}_{1, k}\right\} \\
\operatorname{Cov}\left\{\tilde{\mathbf{H}}_{1, k}^{*}\right\}^{1 / 2}
\end{array}\right], \quad \mathbf{y}_{k} \rightarrow\left[\begin{array}{c}
\mathbf{y}_{k} \\
\mathbf{0}_{R M \times 1}
\end{array}\right]
$$


Furthermore, since the channel response $\mathbf{h}_{2, k}$ is Gaussian, its MAP estimate given the channel vector $\mathbf{h}_{1, k}$ and the output sequence $\mathbf{y}_{k}$ is the same as the MMSE estimate, which can be obtained by the KF. Therefore, given $\mathbf{h}_{1, k}$ and $\mathbf{y}_{k}$, the MAP estimate of $\mathbf{h}_{2, k}$ is obtained by applying the following KF equations to the proposed state model described in (3) and (7), respectively

$$
\begin{gathered}
\hat{\mathbf{h}}_{2, k \mid k-1}=\mathbf{B} \hat{\mathbf{h}}_{2, k-1 \mid k-1} \\
\mathbf{P}_{k \mid k-1}=\mathbf{B} \mathbf{P}_{k-1 \mid k-1} \mathbf{B}^{*}+\sigma_{u}^{2} \mathbf{I}_{M R} \\
\mathbf{K}_{k}=\mathbf{P}_{k \mid k-1} \tilde{\mathbf{H}}_{1, k}^{*}\left(\tilde{\mathbf{H}}_{1, k} \mathbf{P}_{k \mid k-1} \tilde{\mathbf{H}}_{1, k}^{*}+\mathbf{R}_{\mathbf{w}}\right)^{-1} \\
\hat{\mathbf{h}}_{2, k \mid k}=\hat{\mathbf{h}}_{2, k \mid k-1}+\mathbf{K}_{k}\left(\mathbf{y}_{k}-\tilde{\mathbf{H}}_{1, k} \hat{\mathbf{h}}_{2, k \mid k-1}\right) \\
\mathbf{P}_{k \mid k}=\left(\mathbf{I}_{M R}-\mathbf{K}_{k} \tilde{\mathbf{H}}_{1, k}\right) \mathbf{P}_{k \mid k-1} .
\end{gathered}
$$

We can now state the following theorem.

Theorem 1: The channel estimate at the $j$ th iteration $\hat{\mathbf{h}}_{2, k}^{(j)}$ of the EM algorithm is obtained by applying the KF equations (14)-(18) to the following state-space model

$$
\begin{gathered}
\mathbf{h}_{2, k}=\mathbf{B h}_{2, k-1}+\mathbf{u}_{k} \\
{\left[\begin{array}{c}
\mathbf{y}_{k} \\
\mathbf{0}_{R M \times 1}
\end{array}\right]=\left[\begin{array}{c}
E\left\{\tilde{\mathbf{H}}_{1, k}\right\} \\
\operatorname{Cov}\left\{\tilde{\mathbf{H}}_{1, k}^{*}\right\}^{1 / 2}
\end{array}\right] \mathbf{h}_{2, k}+\left[\begin{array}{c}
\mathbf{w}_{k} \\
\mathbf{n}_{k}
\end{array}\right],}
\end{gathered}
$$

where $\mathbf{n}_{k} \in \mathcal{N}\left(\mathbf{0}_{R M \times 1},\left(\sigma_{r}^{2} g_{k} g_{k}^{*}+\sigma_{d}^{2}\right) \mathbf{I}_{R M}\right)$ is virtual noise that is independent from the measurement noise $\mathbf{w}_{k}$.

Remark 1: The conditional mean $E\left\{\tilde{\mathbf{H}}_{1, k}\right\}$ and covariance $\operatorname{Cov}\left\{\tilde{\mathbf{H}}_{1, k}^{*}\right\}$ can be evaluated as

$$
E\left\{\tilde{\mathbf{H}}_{1, k}\right\}=\left(g_{k} E\left\{\mathbf{h}_{1, k}^{T}\right\} \mathbf{X}_{k}\right)^{T} \otimes \mathbf{I}_{M},
$$

$$
\operatorname{Cov}\left\{\tilde{\mathbf{H}}_{1, k}^{*}\right\}=\operatorname{Tr}\left\{\mathbf{X}_{k} \mathbf{X}_{k}^{*}\right\}\left(g_{k} \operatorname{Cov}\left\{\mathbf{h}_{1, k}^{T}\right\} g_{k}^{*}\right) \otimes \mathbf{I}_{M}
$$

Given the most recent estimate $\hat{\mathbf{h}}_{2, k}^{(j-1)}$ and the output sequence $\mathbf{y}_{k}$, the MAP (or equivalently MMSE) estimate of $\mathbf{h}_{1, k}$, and consequently the conditional mean $E\left\{\mathbf{h}_{1, k}\right\}$ and covariance $\operatorname{Cov}\left\{\mathbf{h}_{1, k}\right\}$, can be obtained by applying the KF algorithm to the following state-space model

$$
\begin{gathered}
\mathbf{h}_{1, k}=\mathbf{A h}_{1, k-1}+\mathbf{v}_{k}, \\
\mathbf{y}_{k}=\left(\mathbf{X}_{k}^{T} \otimes \hat{\mathbf{h}}_{2, k}^{(j-1)} g_{k}\right) \mathbf{h}_{1, k}+\mathbf{w}_{k} .
\end{gathered}
$$

\section{B. Extended $K F$}

An alternative approach to jointly estimating the individual channel responses, $\mathbf{h}_{1, k}$ and $\mathbf{h}_{2, k}$, can be obtained by defining the augmented state vector, $\boldsymbol{\theta}_{k} \in \mathcal{C}^{N R+M R \times 1}$ as

$$
\boldsymbol{\theta}_{k}=\left[\begin{array}{ll}
\mathbf{h}_{1, k}^{T} & \mathbf{h}_{2, k}^{T}
\end{array}\right]^{T} .
$$

This leads to a nonlinear state-space model. We can then apply the extended Kalman filter algorithm (EKF) [9] to linearize the problem and estimate the augmented state vector.

After combining the individual state-space equations of (3), we obtain the augmented state-space equation

$$
\boldsymbol{\theta}_{k}=\mathbf{F} \boldsymbol{\theta}_{k-1}+\mathbf{U}_{k},
$$

where $\mathbf{F}=\operatorname{blkdiag}(\mathbf{A}, \mathbf{B})$, and $\mathbf{U}_{k}=\left[\begin{array}{ll}\mathbf{v}_{k}^{T} & \mathbf{u}_{k}^{T}\end{array}\right]^{T}$. The EKF algorithm can be summarized as follows

$$
\begin{gathered}
\hat{\boldsymbol{\theta}}_{k \mid k-1}=\mathbf{F} \hat{\boldsymbol{\theta}}_{k-1 \mid k-1} \\
\mathbf{P}_{k \mid k-1}=\mathbf{F} \mathbf{P}_{k-1 \mid k-1} \mathbf{F}^{*}+\mathbf{Q}_{\mathbf{U}} \\
\mathbf{S}_{k}=\mathbf{J}_{k} \mathbf{P}_{k \mid k-1} \mathbf{J}_{k}^{*}+\mathbf{R}_{\mathbf{w}} \\
\mathbf{K}_{k}=\mathbf{P}_{k \mid k-1} \mathbf{J}_{k}^{*} \mathbf{S}_{k}^{-1} \\
\hat{\boldsymbol{\theta}}_{k \mid k}=\hat{\boldsymbol{\theta}}_{k \mid k-1}+\mathbf{K}_{k}\left(\mathbf{y}_{k}-f\left(\hat{\boldsymbol{\theta}}_{k \mid k-1}\right)\right) \\
\mathbf{P}_{k \mid k}=\left(\mathbf{I}_{(N+M) R}-\mathbf{K}_{k} \mathbf{J}_{k}\right) \mathbf{P}_{k \mid k-1},
\end{gathered}
$$

where $\mathbf{Q}_{\mathbf{U}}=\operatorname{blkdiag}\left(\sigma_{v}^{2} \mathbf{I}_{N R}, \sigma_{u}^{2} \mathbf{I}_{M R}\right)$ is the covariance matrix of the augmented process noise vector, $f\left(\boldsymbol{\theta}_{k}\right)=$ $\operatorname{vec}\left(\mathbf{h}_{2, k} g_{k} \mathbf{h}_{1, k}^{T}\right)$, while $\mathbf{J}_{k}$ is the observation matrix obtained by linearizing the measurement model defined in (7), and (8) around the predicted state

$$
\begin{aligned}
& \mathbf{J}_{k}=\left[\nabla f^{T}(\boldsymbol{\theta})\right]^{T} \mid \hat{\boldsymbol{\theta}}_{k \mid k-1} \\
= & {\left[\begin{array}{ll}
\mathbf{X}_{k}^{T} \otimes g_{k} \hat{\mathbf{h}}_{2, k \mid k-1} & \left(g_{k} \hat{\mathbf{h}}_{1, k \mid k-1}^{T} \mathbf{X}_{k}\right)^{T} \otimes \mathbf{I}_{M}
\end{array}\right] . }
\end{aligned}
$$

The EKF is in general not an optimal estimator and can lead to inconsistent estimates when the linearization errors are not negligible. In such cases, we can further improve the estimation performance by applying the iterated EKF (IEKF) [9]. The state estimate $\hat{\boldsymbol{\theta}}_{k \mid k}^{j}$ at the $j$ th iteration is calculated starting from $\hat{\boldsymbol{\theta}}_{k \mid k-1}$, its uncertainty $\mathbf{P}_{k \mid k-1}$, and the measurement function linearized around $\hat{\boldsymbol{\theta}}_{k \mid k}^{j}$. In summary, after calculating the initial state estimate $\hat{\boldsymbol{\theta}}_{k \mid k}^{1}$, the IEKF algorithm iteratively refines the channel estimates until convergence.

\section{Extension to Multiple Relays}

In this section we extend our analysis to the case of multiple relays $(R>1)$ between the source and the destination. By rewriting the compound channel $\mathbf{H}_{k}=\mathbf{H}_{2, k} \mathbf{G}_{k} \mathbf{H}_{1, k}$ as

$$
\mathbf{H}_{k}=\sum_{i=1}^{R} g_{i, k} \mathbf{h}_{2, k, i} \mathbf{h}_{1, k, i}^{T},
$$


we can obtain the following expression for the measurement equation at the destination

$$
\mathbf{Y}_{k}=\sum_{i=1}^{R}\left(\mathbf{X}^{T} \otimes g_{i, k} \mathbf{h}_{2, k, i}\right) \mathbf{h}_{1, k, i}^{T}+\mathbf{W}_{k}
$$

where the vectors $\mathbf{h}_{1, k, i}$ and $\mathbf{h}_{2, k, i}$ refer to the $i$ th row of $\mathbf{H}_{1, k}$ and the $i$ th column of $\mathbf{H}_{2, k}$, respectively. Since we include $R>1$ relaying nodes, there are $R$ different channel pairs $\mathbf{h}_{1, k, i}, \mathbf{h}_{2, k, i}, i=1, \ldots, R$, to be estimated.

As we show in [12], in order to estimate $\mathbf{H}_{1, k}$ and $\mathbf{H}_{2, k}$, the $k$ th training block should be divided into $R$ frames. For each such frame, we create different compound channels by varying the amplifying factors at the relays. Therefore, by varying the amplifying matrix $\mathbf{G}_{k}$ within $R$ consecutive time slots, we can establish $R$ independent measurements with respect to $\mathbf{H}_{1, k}$ and $\mathbf{H}_{2, k}$ that can guarantee the successful estimation of $\mathbf{h}_{1, k, i}$, $\mathbf{h}_{2, k, i}, i=1, \ldots, R$. The relay amplifying factors during the training block can be designed in such a way so as to reduce the complexity at the relaying nodes [13]. For instance, one could choose $\mathbf{G}_{k}$ such that during the $i$ th estimation interval all but the $i$ th relaying node are switched off. Then, the individual channel pairs $\mathbf{h}_{1, k, i}, \mathbf{h}_{2, k, i}$, can be independently estimated by applying the proposed EM-based KF or the EKF for $i=$ $1, \ldots, R$.

Remark 2: Note that $\mathbf{h}_{1, k, i}$ and $\mathbf{h}_{2, k, i}$ can be determined only up to scaling, since for every $i$ the product $\mathbf{h}_{2, k, i} \mathbf{h}_{1, k, i}$ can be inverted only up to a scalar ambiguity $d_{i}$. Fortunately, this ambiguity causes no problems when optimizing the relay channel because the effect of the $i$ th relay is seen only via the product $\mathbf{h}_{2, i} g_{i, k} \mathbf{h}_{1, i}^{T}$ (see (34)). Moreover, as shown in Section $\mathrm{V}$, the knowledge of $d_{i} \mathbf{h}_{2, k, i}$ and $1 / d_{i} \mathbf{h}_{1, k, i}$ at the destination can be utilized to further improve the overall system performance.

\section{Performance Evaluation}

In this section, we present some numerical results to illustrate the performance of our proposed algorithms. In our simulations a $2 \times 2 \mathrm{MIMO}$ system with $2 \mathrm{AF}$ relaying nodes is considered $(N=M=R=2)$. In each simulation run, the elements of the individual channel matrices $\mathbf{H}_{1, k}$ and $\mathbf{H}_{2, k}$ are generated according to (3), where the elements of $\mathbf{h}_{i, k}$, $i=1,2$, are independent zero-mean circularly symmetric complex Gaussian random variables with unit variance and discrete autocorrelation functions given by (4) and (5). For the sake of simplicity, we use equal normalized Doppler frequencies, i.e., $f_{D 1} T_{s}=f_{D 2} T_{s}=0.005$ that correspond to a moderate fading scenario. The training sequence $\mathbf{X}_{k} \in \mathcal{C}^{N \times L}$ is obtained from the first $N$ rows of a DFT matrix of size $L \times L$. We use the smallest possible value for the length of the training block $L$, that is $L=N=2$. The relay amplifying factors during the estimation phase are generated by using the DFT matrix, scaled in such a way that the relay transmit power constraint is satisfied. We use a fixed transmit power of $0 \mathrm{~dB}$ at the source and at the relays, while we vary the noise power at the relays and the destination. For simplicity, we assume that the noise power is the same at the relays and the destination, i.e. $\sigma_{r}^{2}=\sigma_{d}^{2}$. Simulation results are averaged over
6000 Monte Carlo runs. Due to the ambiguity of the model, the mean-square error (MSE) for the estimation $\mathbf{H}_{1}$ and $\mathbf{H}_{2}$ are defined as

$$
\mathrm{MSE}_{1}=\frac{\left\|\mathbf{H}_{1}-\mathbf{D} \hat{\mathbf{H}}_{1}\right\|_{F}^{2}}{\left\|\mathbf{H}_{1}\right\|_{F}^{2}}, \quad \mathrm{MSE}_{2}=\frac{\left\|\mathbf{H}_{2}-\hat{\mathbf{H}}_{2} \mathbf{D}^{-1}\right\|_{F}^{2}}{\left\|\mathbf{H}_{2}\right\|_{F}^{2}},
$$

where, for simulation purposes, the ambiguity matrix $\mathbf{D}$ is obtained according to [14]

$$
\mathbf{D}=\arg \min _{\mathbf{D}}\left\|\mathbf{H}_{1}-\mathbf{D} \hat{\mathbf{H}}_{1}\right\|_{F}^{2} .
$$

Figure 1 shows the MSE for the estimates of $\mathbf{H}_{1}$ and $\mathbf{H}_{2}$ obtained by the proposed EM-based KF versus the number of iterations, when the SNR during the training interval is equal to $10 \mathrm{~dB}$. It then becomes obvious that the proposed algorithm is numerically stable and that convergence is achieved after 3 iterations. Figure 2 shows the channel estimation performance of the iterated EKF algorithm as a function of the SNR during the training interval. In particular, we illustrate the MSE for $\mathbf{H}_{1}$, for different number of iterations. We observe that the EKF exhibits a sub-optimal performance for a small number of iterations, especially in the high-SNR regime. As we mentioned before, the EKF provides first-order approximations to the optimal terms which may lead to large estimation errors, especially in the case of significant nonlinearities in the measurement function. Moreover, the EKF performance is sensitive to its initialization and can completely lose track if the initial estimates are not accurate. By increasing the number of iterations at the current time step we can improve the estimation performance by effectively redefining the state estimate and re-linearizing the measurement equation. Of course, the application of the IEKF increases the implementation and computational complexity since it requires 9 iterations on the average to converge.

In Figure 3 we compare the performance of the EM-based $\mathrm{KF}$ to the IEKF by plotting the MSE for $\mathbf{H}_{1}$ versus the training SNR when the number of iterations for both filters is equal to 3. We observe that the EM-KF outperforms the EKF since it provides a lower estimation error and does not exhibit an error floor like the EKF, which reaches a floor for SNR $\geq 15 \mathrm{~dB}$. In particular, the two filters exhibit similar tracking performance for low SNR, but the IEKF reaches an error floor at the highSNR regime due to the non-linear measurement model and its sensitivity to initialization errors, whereas the EM-based KF can absorb these discrepancies.

Figure 4 demonstrates the performance improvement when the estimated channels obtained by the proposed EM-KF are utilized to improve signal detection at the destination. In particular, we compare the Zero Forcing (ZF) receiver that requires knowledge of the compound channel to the minimum mean square error (MMSE) receiver which also requires knowledge of the channel link between the relay and the destination $\mathbf{H}_{2}$ for estimating the noise covariance. For this reason, we plot the bit-error-rate (BER) versus the training SNR when the data SNR is equal to $10 \mathrm{~dB}$. The relay gain matrix is chosen proportional to identity. From Figure 4, we observe that the MMSE receiver offers approximately $1 \mathrm{~dB}$ 


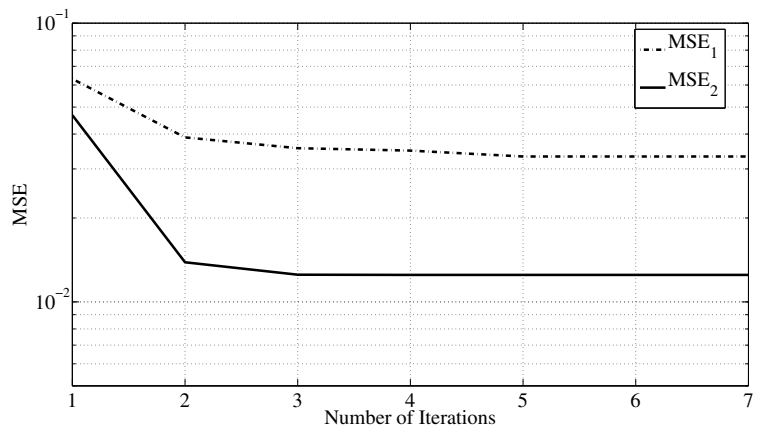

Fig. 1. Channel estimation performance of EM-based KF versus the number of iterations. Training SNR is equal to $10 \mathrm{~dB}$.

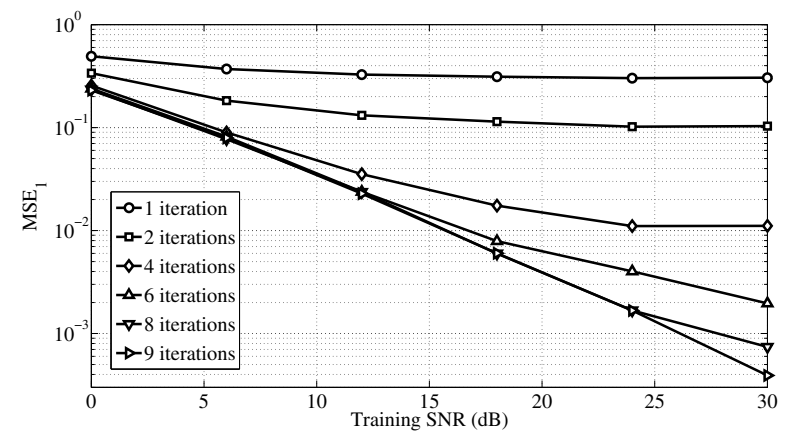

Fig. 2. Channel estimation performance of IEKF for different number of iterations versus training SNR.

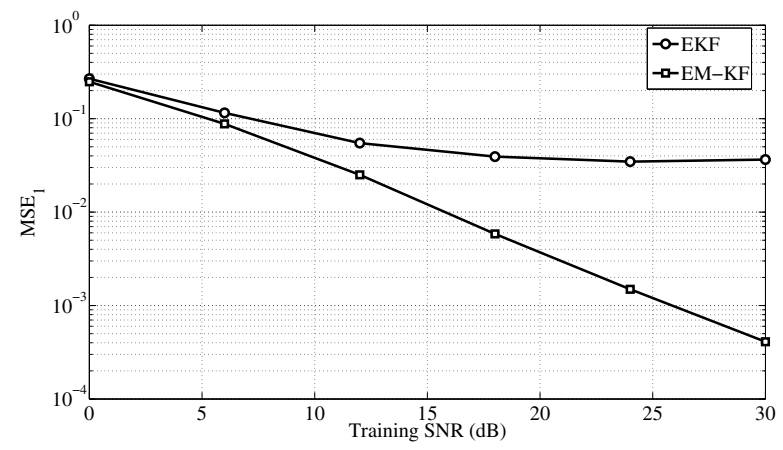

Fig. 3. Comparison of channel estimation performance for $\mathbf{H}_{1}$ between proposed EM-KF and IEKF algorithms. Number of iterations is equal to 3 .

improvement in BER performance when compared to the $\mathrm{ZF}$ receiver. Of course, the performance of both receivers is sensitive to channel estimation errors, but the BER performance improves significantly as the input SNR during the training interval increases.

\section{CONCLUSION}

In this paper, we have investigated the problem of channel estimation in MIMO AF relaying systems with time varying channels. In particular, we developed an expectationmaximization (EM) based Kalman filter that utilizes the received signal at the destination to track the individual channel responses from source to relays and from relays to destination. The extended Kalman filter (EKF) and iterated EKF (IEKF)

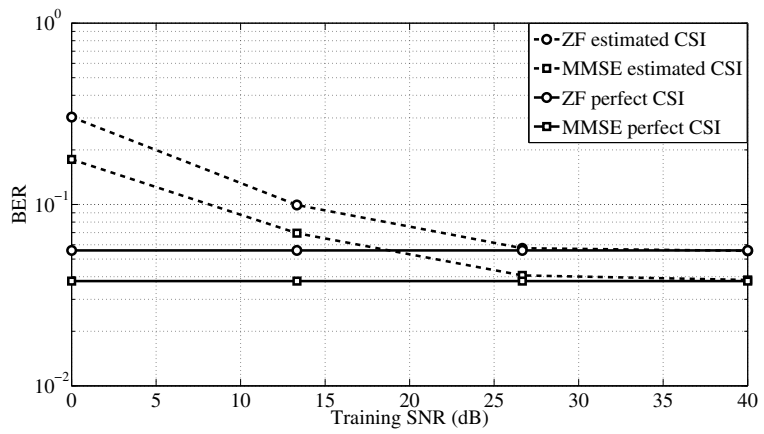

Fig. 4. BER performance comparison between MMSE and ZF receiver versus training SNR. Data SNR $=10 \mathrm{~dB}$.

were also derived and compared to the proposed algorithm. Our theoretical results were supported by simulations for illustrating the effectiveness of the proposed algorithms. Our numerical results showed that the EM-based KF provides better estimation performance with less computational complexity since it converges only after 3 iterations. Finally, we demonstrated the improvement in the BER performance by utilizing the estimated CSI for implementing the MMSE receiver.

\section{REFERENCES}

[1] J. N. Laneman, D. Tse, and G. W. Wornell, "Cooperative diversity in wireless networks: Efficient protocols and outage behavior," IEEE Trans. Inf. Theory, vol. 50, no. 12, pp. 3062-3080, Dec. 2004.

[2] S. Jin, M. R. McKay, C. Zhong, and K. Wong, "Ergodic Capacity Analysis of Amplify-and-Forward MIMO Dual-Hop Systems," IEEE Trans. Inf. Theory, vol. 56, no. 5, pp. 2204 -2224, May 2010.

[3] G. K. Karagiannidis, "Performance bounds of multihop wireless communications with blind relays over generalized fading channels," IEEE Trans. Wireless Commun., vol. 5, no. 3, pp. 498-503, Mar. 2006.

[4] X. Tang and Y. Hua, "Optimal design of non-regenerative MIMO wireless relays," IEEE Trans. Wireless Commun., vol. 6, no. 4, pp. 13981407, Apr. 2007.

[5] F. Gao, T. Cui, and A. Nallanathan, "On channel estimation and optimal training design for amplify and forward relay networks," IEEE Trans. Wireless Commun., vol. 7, no. 5, pp. 1907-1916, May 2008.

[6] P. Lioliou and M. Viberg, "Least-squares based channel estimation for MIMO relays," in Proc. IEEE ITG Workshop on Smart Antennas (WSA), Darmstadt, Germany, Feb. 2008, pp. 90-95.

[7] T. Kong and Y. Hua, "Optimal design of source and relay pilots for MIMO relay channel estimation," IEEE Trans. Signal Process., vol. 59, no. 9, pp. 4438-4446, Sep. 2011.

[8] X. Zhou, T. A. Lamahewa, and P. Sadeghi, "Kalman filter-based channel estimation for amplify and forward relay communications," in Proc. IEEE Asilomar Conf. Signals, Systems, Computers, Pacific Grove, CA, USA, Nov. 2009, pp. 1498-1502.

[9] A. H. Jazwinski, Stochastic Processes and Filtering Theory. New York, Academic Press, 1970.

[10] W. C. Jakes, Microwave Mobile Communications. 2nd ed. Piscataway, NJ: IEEE Press, 1994.

[11] T. Y. Al-Naffouri, "An EM-based forward-backward Kalman filter for the estimation of time-variant channels in OFDM," IEEE Trans. Signal Process., vol. 55, no. 7, pp. 3924-3930, Jul. 2007.

[12] P. Lioliou, M. Viberg, and M. Coldrey, "Performance analysis of relay channel estimation," in Proc. IEEE Asilomar Conf. Signals, Systems, Computers, Pacific Grove, CA, USA, Nov. 2009, pp. 1533-1537.

[13] J. Ma, P. Orlik, J. Zhang, and G. Y. Li, "Pilot matrix design for estimating cascaded channels in two-hop MIMO amplify-and-forward relay systems," IEEE Trans. Wireless Comm., vol. 10, no. 6, pp. 19561965, Jun. 2011.

[14] D. R. Morgan, J. Benesty, and M. M. Sondhi, "On the evaluation of estimated impulse responses," IEEE Signal Process. Lett., vol. 5, no. 7, pp. 174-176, Jul. 1998. 